\title{
New general decay results in an infinite memory viscoelastic problem with nonlinear damping
}

\author{
Adel M. Al-Mahdi ${ }^{* *}$ (1) and Mohammad M. Al-Gharabli
}

\section{*Correspondence:}

almahdi@kfupm.edu.sa

${ }^{1}$ The Preparatory Year Math

Program, King Fahd University of

Petroleum and Minerals, Dhahran,

Saudi Arabia

\begin{abstract}
This work is concerned with a viscoelastic equation with a nonlinear frictional damping and a relaxation function satisfying $g^{\prime}(t) \leq-\xi(t) g^{p}(t), t \geq 0,1 \leq p<\frac{3}{2}$. We establish general decay rate results using the multiplier method and some properties of non-homogeneous ordinary differential inequalities. These results extend and improve many results in the literature.
\end{abstract}

Keywords: General decay; Infinite memory; Viscoelastic problems

\section{Introduction}

In this paper, we consider the following viscoelastic problem:

$$
\begin{cases}u_{t t}-\Delta u+\int_{0}^{+\infty} g(s) \Delta u(t-s) d s+\left|u_{t}\right|^{m-2} u_{t}=0 & \text { in } \Omega \times(0,+\infty), \\ u(x, t)=0 & \text { on } \partial \Omega \times(0,+\infty), \\ u(x,-t)=u_{0}(x, t), u_{t}(x, 0)=u_{1}(x) & \text { in } \Omega \times(0,+\infty),\end{cases}
$$

where $u$ denotes the transverse displacement of waves and $\Omega$ is a bounded domain of $\mathbb{R}^{N}(N \geq 1)$ with a smooth boundary $\partial \Omega, g$ is a positive and decreasing function and $m>1$.

The study of viscoelastic problems has attracted the attention of many authors, and several decay and blow-up results have been established. In [1], Cavalcanti et al. considered the equation

$$
u_{t t}-\Delta u+\int_{0}^{t} g(t-s) \Delta u(x, s) d s+a(x) u_{t}+|u|^{p-1} u=0, \quad \text { in } \Omega \times(0, \infty),
$$

where $a: \Omega \rightarrow \mathbb{R}^{+}$is a function which may vanish on a part of the domain $\Omega$ but satisfies $a(x) \geq a_{0}$ on $\omega \subset \Omega$ and $g$ satisfies, for two positive constants $\xi_{1}$ and $\xi_{2}$,

$$
-\xi_{1} g(t) \leq g^{\prime}(t) \leq-\xi_{2} g(t), \quad t \geq 0
$$

They established an exponential decay result under some restrictions on $\omega$. Berrimi and Messaoudi [2] improved the result of [1], under weaker conditions on both $a$ and $g$, to a

(c) The Author(s) 2019. This article is distributed under the terms of the Creative Commons Attribution 4.0 International License (http://creativecommons.org/licenses/by/4.0/), which permits unrestricted use, distribution, and reproduction in any medium, provided you give appropriate credit to the original author(s) and the source, provide a link to the Creative Commons license, and indicate if changes were made. 
problem where a source term is competing with the damping term. Fabrizio and Polidoro [3] studied the following system:

$$
\begin{cases}u_{t t}-\Delta u+\int_{0}^{t} g(t-\tau) \Delta u(\tau) d \tau+u_{t}=0 & \text { in } \Omega \times(0, \infty) \\ u=0 & \text { on } \partial \Omega \times(0, \infty)\end{cases}
$$

and showed that the exponential decay of the relaxation function is a necessary condition for the exponential decay of the solution energy. Cavalcanti and Oquendo [4] considered the following problem:

$$
u_{t t}-k_{0} \Delta u+\int_{0}^{t} \operatorname{div}[a(x) g(t-s) \Delta u(x, s)] d s+b(x) h\left(u_{t}\right)+f(u)=0
$$

and established, for $a(x)+b(x) \geq \rho>0$, an exponential stability result for $g$ decaying exponentially and $h$ linear and a polynomial stability result for $g$ decaying polynomially and $h$ nonlinear. Rivera [5] considered equations for linear isotropic homogeneous viscoelastic solids of integral type which occupy a bounded domain or the whole space $\mathbb{R}^{n}$, with zero boundary and history data and in the absence of external body forces. In the bounded domain case, an exponential decay result was proved for exponentially decaying memory kernels, and for the whole space case, a polynomial decay result was established and the rate of the decay was given. This latter result was later pushed to a situation where the kernel is decaying algebraically but not exponentially by Cabanillas and Rivera [6]. In their paper, the authors showed that the decay of solutions is also algebraic, at a rate which can be determined by the rate of the decay of the relaxation function and may be improved by the regularity of the initial data. The authors considered both cases, the bounded domains and that of a material occupying the entire space. This result was later improved by Baretto et al. [7], where equations related to linear viscoelastic plates were treated. Precisely, they showed that the solution energy decays at the same decay rate of the relaxation function. For partially viscoelastic materials, Rivera et al. $[8,9]$ showed that solutions decay exponentially to zero, provided the relaxation function decays in a similar fashion, regardless of the size of the viscoelastic part of the material. Pazoto et al. [10] investigated a class of abstract viscoelastic equations of the form

$$
u_{t t}+A u(t)+\beta u(t)-\left(g * A^{\alpha} u\right)(t)=0
$$

for $0 \leq \alpha \leq \beta, \beta \geq 0$. The main focus was on the case when $0<\alpha<1$, and the main result was that solutions for (4) decay polynomially even if the kernel $g$ decays exponentially. This result is sharp (see Theorem 12 [10]). See also Rivera et al. [11], where the authors studied a more general abstract problem than (4) and established a necessary and sufficient condition to obtain an exponential decay. The work of [10] and [11] has been improved by Jamilu and Messaoudi [12].

For infinite history problems, Giorgi et al. [13] considered the following semilinear hyperbolic equation with linear memory in a bounded domain $\Omega \subset \mathbb{R}^{3}$ :

$$
u_{t t}-K(0) \Delta u-\int_{0}^{+\infty} K^{\prime}(s) \Delta u(t-s) d s+g(u)=f, \quad \text { in } \Omega \times \mathbb{R}^{+}
$$


with $K(0), K(\infty)>0$, and $K^{\prime} \leq 0$ and proved the existence of global attractors for the solutions. Conti and Pata [14] considered the following semilinear hyperbolic equation:

$$
u_{t t}+\alpha u_{t}-K(0) \Delta u-\int_{0}^{+\infty} K^{\prime}(s) \Delta u(t-s) d s+g(u)=f, \quad \text { in } \Omega \times \mathbb{R}^{+},
$$

where the memory kernel is a convex decreasing smooth function such that $K(0)>K(\infty)>$ 0 and $g: \mathbb{R} \rightarrow \mathbb{R}$ is a nonlinear term of at most cubic growth satisfying some conditions. They proved the existence of a regular global attractor. In [15], Appleby et al. studied the linear integro-differential equation

$$
u_{t t}+A u(t)+\int_{-\infty}^{t} K(t-s) A u(s) d s=0, \quad t>0
$$

and established an exponential decay result for strong solutions in a Hilbert space. Pata [16] discussed the decay properties of the semigroup generated by the following equation:

$$
u_{t t}+\alpha A u(t)+\beta u_{t}(t)-\int_{0}^{+\infty} \mu(s) A u(t-s) d s=0
$$

where $A$ is a strictly positive self-adjoint linear operator and $\alpha>0, \beta \geq 0$ and the memory kernel $\mu$ is a decreasing function satisfying specific conditions. Subsequently, they established necessary as well as sufficient conditions for the exponential stability. In [17], Guesmia considered

$$
u_{t t}+A u-\int_{0}^{+\infty} g(s) B u(t-s) d s=0
$$

and introduced a new ingenuous approach for proving a more general decay result based on the properties of convex functions and the use of the generalized Young inequality. He used a larger class of infinite history kernels satisfying the following condition:

$$
\int_{0}^{+\infty} \frac{g(s)}{G^{-1}\left(-g^{\prime}(s)\right)} d s+\sup _{s \in \mathbb{R}^{+}} \frac{g(s)}{G^{-1}\left(-g^{\prime}(s)\right)}<+\infty
$$

such that

$$
G(0)=G^{\prime}(0)=0 \text { and } \lim _{t \rightarrow+\infty} G^{\prime}(t)=+\infty
$$

where $G: \mathbb{R}^{+} \rightarrow \mathbb{R}^{+}$is an increasing strictly convex function. Using this approach, Guesmia and Messaoudi [18] later looked into

$$
u_{t t}-\Delta u+\int_{0}^{t} g_{1}(t-s) \operatorname{div}\left(a_{1}(x) \nabla u(s)\right) d s+\int_{0}^{+\infty} g_{2}(s) \operatorname{div}\left(a_{2}(x) \nabla u(t-s)\right) d s=0,
$$

in a bounded domain and under suitable conditions on $a_{1}$ and $a_{2}$ and for a wide class of relaxation functions $g_{1}$ and $g_{2}$ that are not necessarily decaying polynomially or exponentially, and established a general decay result from which the usual exponential and polynomial decay rates are only special cases. Messaoudi and Al-Gharabli [19] considered the 
following nonlinear wave equation:

$$
\left|u_{t}\right|^{\rho} u_{t t}-\Delta u-\Delta u_{t t}+\int_{0}^{+\infty} g(s) \Delta u(t-s) d s=0, \quad \text { in } \Omega \times(0,+\infty)
$$

and proved a general decay result of the solution energy using an approach different from that introduced by Guesmia [17]. For more results in this direction, we refer to [20] and [21].

In the present work, we study the asymptotic behavior of solutions of (1), under assumption (9) (below) instead of (6) considered in Guesmia [17] and Al-Gharabli [22]. This work will extend the result of Belhannache et al. [23] for the finite history case to the infinite history case. The proof of the current result is easier than the one in [17] and [22] since we need no convex function properties or the generalized Young inequality. Moreover, this result gives a better rate of decay in some cases (see Remark 4.3 below).

The rest of this paper is organized as follows. In Sect. 2, we present some assumptions and material needed for our work. Some technical lemmas are presented and proved in Sect. 3. Finally, we state and prove our main decay results and provide some examples in Sect. 4.

\section{Preliminaries}

In this section, we present some materials needed for the proof of our results and state a well-posedness result of the problem. We use the standard Lebesgue space $L^{2}(\Omega)$ and Sobolev space $H_{0}^{1}(\Omega)$ with their usual scalar products and norms and assume the following hypotheses.

(A1) $g: \mathbb{R}^{+} \rightarrow \mathbb{R}^{+}$is a $C^{1}$ nonincreasing function satisfying

$$
g(0)>0, \quad 1-\int_{0}^{+\infty} g(s) d s=\ell>0 .
$$

(A2) There exist a nonincreasing differentiable function $\xi: \mathbb{R}^{+} \rightarrow \mathbb{R}^{+}$and $1 \leq p<\frac{3}{2}$ such that

$$
g^{\prime}(t) \leq-\xi(t) g^{p}(t), \quad \forall t \in \mathbb{R}^{+} .
$$

(A3) For the nonlinearity in the damping, we assume that

$$
1<m \leq \frac{2 n}{n-2}, \quad \text { if } n>2 \quad \text { and } \quad m>1, \quad \text { if } n=1,2 .
$$

(A4) There exists $m_{0} \geq 0$ such that

$$
\left\|\nabla u_{0}(\cdot, s)\right\|_{2} \leq m_{0}, \quad \forall s>0
$$

We introduce the "modified" energy associated to problem (1) by

$$
E(t)=\frac{1}{2}\left\|u_{t}\right\|_{2}^{2}+\frac{1-\ell}{2}\|\nabla u\|_{2}^{2}+\frac{1}{2}(g o \nabla u)(t)
$$


where

$$
(g o \nabla u)(t)=\int_{0}^{+\infty} g(s)\|\nabla u(t)-\nabla u(t-s)\|_{2}^{2} d s .
$$

Direct differentiation, using (1), leads to

$$
E^{\prime}(t)=\frac{1}{2}\left(g^{\prime} o \nabla u\right)(t)-\int_{\Omega}\left|u_{t}\right|^{m} d x \leq 0 .
$$

Now, we state without proof the existence result to problem (1).

Proposition $1([22,23])$ Let $\left(u_{0}(\cdot, 0), u_{1}\right) \in H_{0}^{1}(\Omega) \times L^{2}(\Omega)$ be given. Assume that $(A 1)-$ (A4) hold and $m>1$. Then problem (1) has a unique weak global solution.

\section{Technical lemmas}

In this section, we state and establish several lemmas needed for the proof of our main result.

Lemma 3.1 ([4, 24]) Assume that $g$ satisfies $(A 1)$ and (A2), then

$$
\int_{0}^{+\infty} \xi(t) g^{1-\sigma}(t) d t<+\infty, \quad \forall \sigma<2-p
$$

Lemma $3.2([4,24])$ Assume that (A1) and (A2) hold and $u$ is the solution of (1) then, for $0<\sigma<1$, we have

$$
\int_{0}^{t} g(s)\|\nabla u(t-s)-\nabla u(t)\|_{2}^{2} d s \leq c\left[\left(\int_{0}^{t} g^{1-\sigma}(t) d t\right) E(0)\right]^{\frac{p-1}{p-1+\sigma}}\left(g^{p} o \nabla u\right)^{\frac{\sigma}{p-1+\sigma}}
$$

By taking $\sigma=\frac{1}{2}$, we get

$$
\int_{0}^{t} g(s)\|\nabla u(t-s)-\nabla u(t)\|_{2}^{2} d s \leq c\left[\int_{0}^{t} g^{\frac{1}{2}}(s) d s\right]^{\frac{2 p-2}{2 p-1}}\left(g^{p} o \nabla u\right)^{\frac{1}{2 p-1}}(t) .
$$

Remark 3.1 Using (12), (A4), and the fact $E$ is nonincreasing, we obtain

$$
\begin{aligned}
\|\nabla u(t)-\nabla u(t-s)\|_{2}^{2} \\
\quad \leq 2\|\nabla u(t)\|_{2}^{2}+2\|\nabla u(t-s)\|_{2}^{2} \\
\quad \leq 4 \sup _{s>0}\|\nabla u(s)\|_{2}^{2}+2 \sup _{\tau<0}\|\nabla u(\tau)\|_{2}{ }^{2} \\
\quad \leq 4 \sup _{s>0}\|\nabla u(s)\|_{2}^{2}+2 \sup _{\tau>0}\left\|\nabla u_{0}(\tau)\right\|_{2}^{2} \\
\quad \leq \frac{8}{1-\ell} E(0)+2 m_{0}^{2}:=N_{1} .
\end{aligned}
$$

Corollary 1 Assume that (A1)-(A4) hold and $u$ is a solution of (1), then

$$
\xi(t) \int_{0}^{+\infty} g(s)\|\nabla u(t-s)-\nabla u(t)\|_{2}^{2} d s \leq C\left[-E^{\prime}(t)\right]^{\frac{1}{2 p-1}}+N_{1} \xi(t) \int_{t}^{+\infty} g(s) d s .
$$


Proof Multiply both sides of (15) by $\xi(t)$ and use (13), (14), and (16) to obtain

$$
\begin{aligned}
\xi(t) & \int_{0}^{+\infty} g(s)\|\nabla u(t-s)-\nabla u(t)\|_{2}^{2} d s \\
& =\xi(t) \int_{0}^{t} g(s)\|\nabla u(t-s)-\nabla u(t)\|_{2}^{2} d s+\xi(t) \int_{t}^{+\infty} g(s)\|\nabla u(t-s)-\nabla u(t)\|_{2}^{2} d s \\
& \leq C \xi^{\frac{2 p-2}{2 p-1}}(t)\left[\int_{0}^{t} g^{\frac{1}{2}}(s) d s\right]^{\frac{2 p-2}{2 p-1}} \xi^{\frac{1}{2 p-1}}(t)\left(g^{p} o \nabla u\right)^{\frac{1}{2 p-1}}(t)+N_{1} \xi(t) \int_{t}^{+\infty} g(s) d s \\
& \leq C\left[\int_{0}^{t} \xi(s) g^{\frac{1}{2}}(s) d s\right]^{\frac{2 p-2}{2 p-1}}\left(\xi g^{p} o \nabla u\right)^{\frac{1}{2 p-1}}(t)+N_{1} \xi(t) \int_{t}^{+\infty} g(s) d s \\
& \leq C\left[\int_{0}^{+\infty} \xi(s) g^{\frac{1}{2}}(s) d s\right]^{\frac{2 p-2}{2 p-1}}\left(-g^{\prime} o \nabla u\right)^{\frac{1}{2 p-1}}(t)+N_{1} \xi(t) \int_{t}^{+\infty} g(s) d s \\
& \leq C\left[-E^{\prime}(t)\right]^{\frac{1}{2 p-1}}+N_{1} \xi(t) \int_{t}^{+\infty} g(s) d s .
\end{aligned}
$$

Lemma 3.3 ([22]) Under assumptions $(A 1)-(A 4)$, the functional

$$
\psi(t):=\int_{\Omega} u u_{t} d x
$$

satisfies, along the solution, the estimate

$$
\begin{aligned}
\psi^{\prime}(t) \leq & -\frac{\ell}{4}\|\nabla u\|_{2}^{2}+\left\|u_{t}\right\|_{2}^{2}+\frac{1-\ell}{2 \ell}(g o \nabla u)(t) \\
& +c \int_{\Omega}\left|u_{t}\right|^{m} d x, \quad \text { if } m \geq 2
\end{aligned}
$$

and

$$
\begin{aligned}
\psi^{\prime}(t) \leq & -\frac{\ell}{4}\|\nabla u\|_{2}^{2}+\left\|u_{t}\right\|_{2}^{2}+\frac{1-\ell}{2 \ell}(g o \nabla u)(t) \\
& +c(\Omega)\left(\int_{\Omega}\left|u_{t}\right|^{m} d x\right)^{\frac{2 m-2}{m}}, \quad \text { if } m<2 .
\end{aligned}
$$

Lemma $3.4([22])$ Under assumptions $(A 1)-(A 4)$, the functional

$$
\chi(t):=-\int_{\Omega} u_{t} \int_{0}^{+\infty} g(s)(u(t)-u(t-s)) d s d x
$$

satisfies, for all $\delta>0$ and along the solution, the estimate

$$
\begin{aligned}
\chi^{\prime}(t) \leq & -\delta\left[1+2(1-\ell)^{2}\right]\|\nabla u\|_{2}^{2}-((1-\ell)-\delta)\left\|u_{t}\right\|_{2}^{2}+C(\delta)(g o \nabla u)(t) \\
& +\frac{g(0)}{4 \delta}\left(-\left(g^{\prime} o \nabla u\right)\right)(t)+C(\delta) \int_{\Omega}\left|u_{t}\right|^{m} d x, \quad \text { if } m \geq 2
\end{aligned}
$$

and

$$
\chi^{\prime}(t) \leq-\delta\left[1+2(1-\ell)^{2}\right]\|\nabla u\|_{2}^{2}-((1-\ell)-\delta)\left\|u_{t}\right\|_{2}^{2}+C(\delta)(g o \nabla u)(t)
$$




$$
+\frac{g(0)}{4 \delta}\left(-\left(g^{\prime} o \nabla u\right)\right)(t)+c(\delta, \Omega)\left(\int_{\Omega}\left|u_{t}\right|^{m} d x\right)^{\frac{2 m-2}{m}}, \quad \text { if } m<2 .
$$

Lemma 3.5 ([22]) Assume that (A1)-(A4) hold. Then there exist strictly positive constants $\varepsilon_{1}, \varepsilon_{2}, \alpha_{1}, c$ such that the functional

$$
L=E(t)+\varepsilon_{1} \psi(t)+\varepsilon_{2} \chi(t)
$$

satisfies, for all $t \in \mathbb{R}^{+}$,

$$
\begin{aligned}
& F \sim E, \\
& L^{\prime}(t) \leq-\alpha_{1} E(t)+c(g o \nabla u)(t) \quad \text { if } m \geq 2
\end{aligned}
$$

and

$$
L^{\prime}(t) \leq-\alpha_{1} E(t)+c(g o \nabla u)(t)+c\left(\int_{\Omega}\left|u_{t}\right|^{m} d x\right)^{\frac{2 m-2}{m}} \quad \text { if } m<2 .
$$

\section{The main result}

In this section we state and prove our decay result. We start with two remarks.

Remark 4.1 If $1+\frac{1}{4 p-3}<m<2$, we have

$$
\frac{2 m-2}{m}>\frac{1}{2 p-1}
$$

and if $1<m<1+\frac{1}{4 p-3}<2$, we have

$$
\frac{2 m-2}{m}<\frac{1}{2 p-1}
$$

Remark 4.2 Using (13) and (16), we have

$$
\left|E^{\prime}(t)\right| \leq \frac{1}{2}\left|g^{\prime} o \nabla u(t)\right| \leq \frac{N_{1}}{2} g(0)=c .
$$

Theorem 4.1 Let $\left(u_{0}(\cdot, 0), u_{1}\right) \in H_{0}^{1}(\Omega) \times L^{2}(\Omega)$ be given. Assume that $(A 1)-(A 4)$ hold. Then, for $m \geq 2$, we have

$$
E(t) \leq \delta_{1}\left(1+\int_{0}^{t}(g(s))^{1-\delta_{0}} d s\right) e^{-\delta_{0} \int_{0}^{t} \xi(s) d s}+\delta_{1} \int_{t}^{+\infty} g(s) d s, \quad p=1,
$$

and

$$
E(t) \leq C(1+t)^{\frac{-1}{2 p-2}} \xi^{-\frac{2 p-1}{2 p-2}}(t)\left(1+\int_{0}^{t}(1+s)^{\frac{1}{2 p-2}} \xi^{\frac{2 p-1}{2 p-2}}(s) h^{2 p-1} d s\right), \quad 1<p<\frac{3}{2} .
$$

Moreover, for any $1<p<\frac{3}{2}$, if

$$
\int_{0}^{+\infty}\left[(1+t)^{\frac{-1}{2 p-2}} \xi^{-\frac{2 p-1}{2 p-2}}(t)\left(1+\int_{0}^{t}(1+s)^{\frac{1}{2 p-2}} \xi^{\frac{2 p-1}{2 p-2}}(s) h^{2 p-1}(s) d s\right)\right] d t<+\infty
$$


then we have

$$
E(t) \leq C(1+t)^{\frac{-1}{p-1}} \xi^{-\frac{2 p}{p-1}}(t)\left(1+\int_{0}^{t}(1+s)^{\frac{1}{p-1}} \xi^{\frac{p}{p-1}}(s) h^{p}(s) d s\right), \quad 1<p<\frac{3}{2},
$$

where $\left.C h(t):=N_{1} \xi(t) \int_{t}^{+\infty} g(s) d s\right), \delta_{1}, C$ are strictly positive numbers and $\delta_{0} \in\left(0, \gamma_{0}\right], \gamma_{0} \in$ $(0,1)$.

Remark 4.3 Let us compare our estimates (30) and (32) with the one of [17] and [22] obtained for (1). Our estimate (32) improves the decay rate given in [17]. Indeed, let $g(t)=\frac{a}{(1+t)^{q}}, q>2$, where $a$ is chosen so that hypothesis $(A 1)$ remains valid. Then

$$
g^{\prime}(t)=\frac{-a q}{(1+t)^{q+1}}=-b\left(\frac{a}{(1+t)^{q}}\right)^{\frac{q+1}{q}}=-b g^{p}(t), \quad p=\frac{q+1}{q}<\frac{3}{2}, b>0 .
$$

Let us compute

$$
h(t)=\xi(t) \int_{t}^{+\infty} g(s) d s=\frac{a b}{q-1}(1+t)^{1-q}, \quad q=\frac{1}{p-1} .
$$

Routine calculations yield, for some positive constant $C$,

$$
\int_{0}^{t}(1+s)^{\frac{1}{p-1}} \xi^{\frac{p}{p-1}}(s) h^{p} d s=C(1+t)^{p(1-q)+\frac{1}{p-1}+1}-C .
$$

Therefore, estimate (32) yields

$$
E(t) \leq C(1+t)^{\frac{-q^{2}+q+1}{q}},
$$

which implies that (36) improves the following decay rate obtained in [17]:

$$
E(t) \leq C(1+t)^{-p}, \quad \forall 0<p<\frac{q-1}{2} .
$$

This is because $\frac{q^{2}-q-1}{2}>\frac{q-1}{2}$ for $q>2$. As a conclusion our approach improves and has a better decay rate than the one of [17].

Proof of Theorem 4.1 For the proof of (29), see [19]. For (30), we multiply (24) by $\xi^{\alpha+1}(t) E^{\alpha}(t)$, where $\alpha=2 p-2$, and use (17) to obtain

$$
\xi^{\alpha+1}(t) E^{\alpha}(t) L^{\prime}(t) \leq-\alpha_{1} \xi^{\alpha+1}(t) E^{\alpha+1}(t)+c(\xi E)^{\alpha}(t)\left[-E^{\prime}(t)\right]^{\frac{1}{\alpha+1}}+C h(t) \xi^{\alpha}(t) E^{\alpha}(t) .
$$

Use of Young's inequality, with $q=\alpha+1$ and $q^{*}=\frac{\alpha+1}{\alpha}$, gives

$$
\xi^{\alpha+1}(t) E^{\alpha}(t) L^{\prime}(t) \leq-\alpha_{1} \xi^{\alpha+1}(t) E^{\alpha+1}(t)+2 \varepsilon \xi^{\alpha+1}(t) E^{\alpha+1}-C_{\varepsilon} E^{\prime}(t)+C_{\varepsilon} h^{\alpha+1}(t) .
$$

Choosing $\varepsilon$ small enough and letting $F:=\xi^{\alpha+1} E^{\alpha} L+C_{\varepsilon} E \sim E$, we have, for positive constants $c_{1}$ and $c_{2}$,

$$
F^{\prime}(t) \leq-c_{1} \xi^{\alpha+1}(t) F^{\alpha+1}(t)+c_{2} h^{\alpha+1}(t)
$$


Multiplying both sides of (40) by $\xi^{\beta}, \beta>1$, we get

$$
\xi^{\beta} F^{\prime}(t) \leq-c_{1} \xi^{\alpha+1+\beta}(t) F^{\alpha+1}(t)+c_{2} \xi^{\beta} h^{\alpha+1}(t) .
$$

Recalling that $\xi>0$ and nonincreasing, one can see that

$$
\text { \%labelL0 }\left(\xi^{\beta} F(t)\right)^{\prime} \leq-c_{1} \xi^{\alpha+1+\beta}(t) F^{\alpha+1}(t)+c_{2} \xi^{\beta} h^{\alpha+1}(t) .
$$

Noting $\varphi=\xi^{\beta} F$ and taking $\beta=\frac{\alpha+1}{\alpha}$, we obtain

$$
\varphi^{\prime}(t) \leq-c_{1} \varphi^{\alpha+1}(t)+c_{2} \xi^{\beta}(t) h^{\alpha+1}(t)
$$

Let

$$
f(t):=\varphi(t)-\Psi(t) ; \quad \text { where } \Psi(t)=c_{2}(1+t)^{\frac{-1}{\alpha}} \int_{0}^{t} \xi^{\beta}(s) h^{\alpha+1}(s)(1+s)^{\frac{1}{\alpha}} d s .
$$

From the definition of $\Psi$, we have

$$
c_{2} \xi^{\beta}(t) h^{\alpha+1}(t)=\Psi^{\prime}(t)+\frac{c_{2}}{\alpha}(1+t)^{\frac{-1}{\alpha}-1} \int_{0}^{t} \xi^{\beta}(s) h^{\alpha+1}(s)(1+s)^{\frac{1}{\alpha}} d s .
$$

Since $\xi^{\beta}(s) h^{\alpha+1}(1+s)^{\frac{1}{\alpha}}>0$, we have, for all $t \geq t_{0}>0$,

$$
v:=\int_{0}^{t_{0}} \xi^{\beta}(s) h^{\alpha+1}(s)(1+s)^{\frac{1}{\alpha}} d s \leq \int_{0}^{t} \xi^{\beta}(s) h^{\alpha+1}(s)(1+s)^{\frac{1}{\alpha}} d s
$$

and then

$$
\frac{\int_{0}^{t} \xi^{\beta}(s) h^{\alpha+1}(s)(1+s)^{\frac{1}{\alpha}} d s}{v} \geq 1, \quad \forall t \geq t_{0} .
$$

Thus (45) yields, $\forall t \geq t_{0}$,

$$
c_{2} \xi^{\beta}(t) h^{\alpha+1}(t) \leq \Psi^{\prime}(t)+\frac{1}{\alpha c_{2}^{\alpha} \nu^{\alpha}} c_{2}^{\alpha+1}\left[(1+t)^{\frac{-1}{\alpha}}\right]^{\alpha+1}\left[\int_{0}^{t} \xi^{\beta}(s) h^{\alpha+1}(s)(1+s)^{\frac{1}{\alpha}} d s\right]^{\alpha+1} .
$$

We can choose $c_{2}$ large enough so that $\frac{1}{\alpha c_{2}^{\alpha} \nu^{\alpha}} \leq c_{1}$, and then we get

$$
c_{2} \xi^{\beta}(t) h^{\alpha+1}(t) \leq \Psi^{\prime}(t)+c_{1} \Psi^{\alpha+1}, \quad \forall t \geq t_{0} .
$$

Now, using (47) and the definition of $f$, we get, $\forall t \geq t_{0}$,

$$
\begin{aligned}
f^{\prime}(t)=\varphi^{\prime}(t)-\Psi^{\prime}(t) & \leq-c_{1} \varphi^{\alpha+1}(t)+c_{2} \xi^{\beta}(t) h^{\alpha+1}(t)-\Psi^{\prime}(t) \\
& \leq-c_{1}\left[(f+\Psi)^{\alpha+1}(t)\right]+c_{2} \xi^{\beta}(t) h^{\alpha+1}(t)-\Psi^{\prime}(t)
\end{aligned}
$$

Since $f(0)>0$, then there exists $t_{1}>0$ such that $f(t)>0, \forall t \in\left[0, t_{1}\right)$. Hence,

$$
f^{\prime}(t) \leq-c_{1}\left[f^{\alpha+1}(t)+\Psi^{\alpha+1}(t)\right]+c_{2} \xi^{\beta}(t) h^{\alpha+1}(t)-\Psi^{\prime}(t), \quad \forall t \in\left[t_{0}, t_{1}\right)
$$




$$
\leq-c_{1}\left[f^{\alpha+1}(t)+\Psi^{\alpha+1}(t)-\frac{c_{2}}{c_{1}} \xi^{\beta}(t) h^{\alpha+1}(t)+\frac{1}{c_{1}} \Psi^{\prime}(t)\right] .
$$

Thus,

$$
f^{\prime}(t) \leq-c_{1} f^{\alpha+1}(t), \quad \forall t \in\left[t_{0}, t_{1}\right)
$$

Integrating over $\left(t_{0}, t\right)$, we have

$$
f(t) \leq \frac{c}{\left(t-t_{0}\right)^{\frac{1}{\alpha}}}, \quad \forall t \in\left[t_{0}, t_{1}\right)
$$

If $t_{1}=+\infty$, using again the definitions of $f$ and $\Psi$, we have, for $t$ large enough,

$$
\varphi(t) \leq C(1+t)^{\frac{-1}{\alpha}}\left[1+\int_{0}^{t} \xi^{\beta}(s) h^{\alpha+1}(s)(1+s)^{\frac{1}{\alpha}} d s\right] .
$$

If $t_{1}<+\infty$, then there exists $t_{2}>t_{1}$ such that $f(t) \leq 0, \forall t_{1} \leq t<t_{2}$. Hence, (44) yields $\varphi(t) \leq$ $\Psi(t), \forall t_{1} \leq t<t_{2}$; consequently, we get (52). If $t_{2}=+\infty$, we are done. Otherwise, there exists $t_{3}>t_{2}$ such that $f\left(t_{2}\right)=0$ and $f(t)>0, \forall t_{2}<t<t_{3}$, we then repeat steps (49)-(51) on $\left[t_{2}, t_{3}\right)$ to obtain (52). Therefore, (52) remains valid for all $t \geq t_{0}$. Multiply (52) by $\xi^{-\beta}$ and recall the definition of $\varphi$, then for $\beta=\frac{\alpha+1}{\alpha}$ we have

$$
F(t) \leq C(1+t)^{\frac{-1}{\alpha} \xi^{-\frac{\alpha+1}{\alpha}}}\left[1+\int_{0}^{t} \xi^{\frac{\alpha+1}{\alpha}}(s) h^{\alpha+1}(s)(1+s)^{\frac{1}{\alpha}} d s\right] .
$$

Using the fact $F \sim E$ and recalling that $\alpha=2 p-2$, we get

$$
E(t) \leq C(1+t)^{\frac{-1}{2 p-2}} \xi^{-\frac{2 p-1}{2 p-2}}\left(1+\int_{0}^{t}(1+s)^{\frac{1}{2 p-2}} \xi^{\frac{2 p-1}{2 p-2}}(s) h^{2 p-1} d s\right) .
$$

This establishes (30).

To show (32), we note that simple calculations, using (30) and (31), yield

$$
\int_{t_{0}}^{+\infty} E(t) d t<+\infty
$$

Use (55) in the following quantity to obtain

$$
\begin{aligned}
I(t) & :=\int_{0}^{t}\|\nabla u(t-s)-\nabla u(t)\|_{2}^{2} d s \leq C \int_{0}^{t}\left(\|\nabla u(t-s)\|_{2}^{2}+\|\nabla u(t)\|_{2}^{2}\right) d s \\
& \leq C \int_{0}^{t}[E(t-s)+E(t)] d s \\
& \leq 2 C \int_{0}^{t} E(t-s) d s \\
& \leq 2 C \int_{0}^{t} E(s) d s<2 C \int_{0}^{\infty} E(s) d s<\infty .
\end{aligned}
$$


Without loss of the generality, we assume that $I(t)>0$ for all $t \geq t_{0}$; otherwise (1) yields an exponential decay. Assumption (A2), Jensen's inequality, and the fact that $\xi$ is nonincreasing lead to

$$
\begin{aligned}
& \xi(t) \int_{0}^{t} g(s)\|\nabla u(t-s)-\nabla u(t)\|_{2}^{2} d s \\
& \leq \frac{I(t)}{I(t)} \int_{0}^{t}\left(\xi^{p}(s) g^{p}(s)\right)^{\frac{1}{p}}\|\nabla u(t-s)-\nabla u(t)\|_{2}^{2} d s \\
& \leq C I(t)\left(\frac{1}{I(t)} \int_{0}^{t} \xi^{p}(s) g^{p}(s)\|\nabla u(t-s)-\nabla u(t)\|_{2}^{2} d s\right)^{\frac{1}{p}} \\
& \leq C I^{1-\frac{1}{p}}(t)\left(\int_{0}^{t} \xi^{p}(s) g^{p}(s)\|\nabla u(t-s)-\nabla u(t)\|_{2}^{2} d s\right)^{\frac{1}{p}} \\
& \leq C I^{1-\frac{1}{p}}(t) \xi^{p-1}(0)\left(\int_{0}^{t} \xi(s) g^{p}(s)\|\nabla u(t-s)-\nabla u(t) b i g r\|_{2}^{2} d s\right)^{\frac{1}{p}} \\
& \leq C\left(\int_{0}^{t}-g^{\prime}(s)\|\nabla u(t-s)-\nabla u(t)\|_{2}^{2} d s\right)^{\frac{1}{p}} \\
& \leq C\left(-E^{\prime}(t)\right)^{\frac{1}{p}} \text {. }
\end{aligned}
$$

Multiply (24) by $\xi(t)$ to get

$$
\begin{aligned}
\xi(t) L^{\prime}(t) \leq & -\alpha_{1} \xi(t) E(t)+\alpha_{2} \xi(t) \int_{0}^{t} g(s)\|\nabla u(t)-\nabla u(t-s)\|_{2}^{2} d s \\
& +\alpha_{2} \xi(t) \int_{t}^{+\infty} g(s)\|\nabla u(t)-\nabla u(t-s)\|_{2}^{2} d s .
\end{aligned}
$$

Then, using (57), (16), and the definition of $C h(t)$, we have

$$
\xi(t) L^{\prime}(t) \leq-\alpha_{1} \xi(t) E(t)+C\left[-E^{\prime}(t)\right]^{\frac{1}{p}}+C h(t) .
$$

Multiply (59) by $\xi^{\alpha}(t) E^{\alpha}(t)$, where $\alpha=p-1$, to obtain

$$
\begin{aligned}
& \xi^{\alpha+1}(t) E^{\alpha}(t) L^{\prime}(t) \\
& \quad \leq-\alpha_{1} \xi^{\alpha+1}(t) E^{\alpha+1}(t)+C(\xi E)^{\alpha}(t)\left[-E^{\prime}(t)\right]^{\frac{1}{\alpha+1}}+C h(t) \xi^{\alpha}(t) E^{\alpha}(t) .
\end{aligned}
$$

Use of Young's inequality, with $q=\alpha+1$ and $q^{*}=\frac{\alpha+1}{\alpha}$, gives

$$
\xi^{\alpha+1}(t) E^{\alpha}(t) L^{\prime}(t) \leq-\alpha_{1} \xi^{\alpha+1}(t) E^{\alpha+1}(t)+2 \varepsilon \xi^{\alpha+1}(t) E^{\alpha+1}-C_{\varepsilon} E^{\prime}(t)+C_{\varepsilon} h^{\alpha+1}(t) .
$$

Choose $\varepsilon$ small enough and let $\widetilde{F}:=\xi^{\alpha+1} E^{\alpha} L+C_{\varepsilon} E \sim E$, then there exist positive constants $\beta_{1}$ and $\beta_{2}$ such that

$$
\widetilde{F}^{\prime}(t) \leq-\beta_{1} \xi^{\alpha+1}(t) \widetilde{F}^{\alpha+1}(t)+\beta_{2} h^{\alpha+1}(t) .
$$


Repeating the same computations as above, we obtain

$$
E(t) \leq C(1+t)^{\frac{-1}{p-1}} \xi^{-\frac{2 p}{p-1}}\left(1+\int_{0}^{t}(1+s)^{\frac{1}{p-1}} \xi^{\frac{p}{p-1}}(s) h^{p} d s\right)
$$

This establishes (32).

Theorem 4.2 Let $\left(u_{0}(\cdot, 0), u_{1}\right) \in H_{0}^{1}(\Omega) \times L^{2}(\Omega)$ be given. Assume that $(A 1)-(A 4)$ hold. Then, for $1<m<2, p=1$, and positive constants $c_{i}, i=1,2,3$, we have the following estimate:

$$
E(t) \leq c_{1} e^{-\delta_{0} \int_{0}^{t} \xi(s) d s}\left(c_{2}+c_{3} \int_{0}^{t} e^{\delta_{0} \int_{0}^{t} \xi(s) d s} H(s) d s\right),
$$

where $H(t)=h(t)+\varepsilon \xi^{\frac{m}{2-m}}(t)$.

Proof For (64), we multiply (25) by $\xi(t)$; using (13), (17), and Young's inequality, we have

$$
\begin{aligned}
\xi(t) L^{\prime}(t) & \leq-\alpha_{1} \xi(t) E(t)+c\left[-E^{\prime}(t)\right]+C h(t)+c \xi(t)\left(\int_{\Omega}\left|u_{t}\right|^{m} d x\right)^{\frac{2 m-2}{m}} \\
& \leq-\alpha_{1} \xi(t) E(t)+c\left[-E^{\prime}(t)\right]+C h(t)+c \xi(t)\left(-E^{\prime}(t)\right)^{\frac{2 m-2}{m}} \\
& \leq-\alpha_{1} \xi(t) E(t)+C h(t)+\varepsilon \xi^{\frac{m}{2-m}}(t)-(c(\varepsilon)+c) E^{\prime}(t) .
\end{aligned}
$$

By letting $\mathbb{F}(t):=\xi(t) L(t)+(c(\varepsilon)+c) E(t) \sim E(t)$, we arrive at

$$
\mathbb{F}^{\prime}(t) \leq-\alpha_{1} \xi(t) \mathbb{F}(t)+C H(t)
$$

where $H(t)=h(t)+\varepsilon \xi^{\frac{m}{2-m}}(t)$. Repeating the same steps of [25], then (64) is established.

Remark 4.4 Estimate (64) gives a decay estimate on $E(t)$ if $\xi(t)$ converges to zero when $t$ goes to infinity. If $\xi(t)$ is a constant, that is, $g^{\prime}(t) \leq-\xi g(t)$, then $g(t)$ converges to zero exponentially when $t$ goes to infinity. In this case, we have the following estimates:

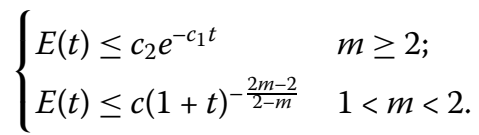

For the proof, see Theorem 4.1 in [22].

Theorem 4.3 Let $\left(u_{0}(\cdot, 0), u_{1}\right) \in H_{0}^{1}(\Omega) \times L^{2}(\Omega)$ be given. Assume that $(A 1)-(A 4)$ hold. Then we have, for $1<m<2$ and $1<p<\frac{3}{2}$, the following estimates:

$$
\begin{aligned}
E(t) & \leq C(1+t)^{\frac{-1}{2 p-2}} \xi^{-\frac{2 p-1}{2 p-2}}(t)\left(1+\int_{0}^{t}(1+s)^{\frac{1}{2 p-2}} \xi^{\frac{2 p-1}{2 p-2}}(s) h^{2 p-1}(s) d s\right) \\
1 & +\frac{1}{4 p-3}<m<2
\end{aligned}
$$


and

$$
\begin{aligned}
E(t) & \leq C(1+t)^{-\frac{2 m-2}{m-2}} \xi^{-\frac{m}{2-m}}(t)\left(1+\int_{0}^{t}(1+s)^{\frac{2 m-2}{m-2}} \xi^{\frac{m}{2-m}}(s) h^{\frac{m}{2 m-2}}(s) d s\right), \\
1 & <m<1+\frac{1}{4 p-3} .
\end{aligned}
$$

Moreover, if $1<m<1+\frac{1}{4 p-3}$ and

$$
\int_{0}^{+\infty}\left[(1+t)^{-\frac{2 m-2}{m-2}} \xi^{-\frac{m}{2-m}}(t)\left(1+\int_{0}^{t}(1+s)^{\frac{2 m-2}{m-2}} \xi^{\frac{m}{2-m}}(s) h^{\frac{m}{2 m-2}}(s) d s\right)\right]<+\infty
$$

then

$$
E(t) \leq C(1+t)^{-\frac{4 m-4}{2-m}} \xi^{-\frac{6 m-4}{2-m}}(t)\left(1+\int_{0}^{t}(1+s)^{\frac{4 m-4}{2-m}} \xi^{\frac{6 m-4}{2-m}}(s) h^{\frac{3 m-2}{4 m-4}}(s) d s\right)
$$

where $h(t)=\xi(t) \int_{t}^{\infty} g(s) d s$ and $C$ is a positive constant.

Proof For (68), we multiply (25) by $\xi^{\alpha+1}(t) E^{\alpha}(t)$, where $\alpha=2 p-2$. Recall the definition of $h(t)$ and use (17) to obtain

$$
\begin{aligned}
\xi^{\alpha+1}(t) E^{\alpha}(t) L^{\prime}(t) \leq & -\alpha_{1} \xi^{\alpha+1}(t) E^{\alpha+1}(t)+c(\xi E)^{\alpha}(t)\left[-E^{\prime}(t)\right]^{\frac{1}{2 p-1}} \\
& +C h(t) \xi^{\alpha}(t) E^{\alpha}(t)+c \xi(t)(\xi E)^{\alpha}(t)\left(\int_{\Omega}\left|u_{t}\right|^{m} d x\right)^{\frac{2 m-2}{m}}
\end{aligned}
$$

Then exploit (13) to get

$$
\begin{aligned}
\xi^{\alpha+1}(t) E^{\alpha}(t) L^{\prime}(t) \leq & -\alpha_{1} \xi^{\alpha+1}(t) E^{\alpha+1}(t)+c(\xi E)^{\alpha}(t)\left[-E^{\prime}(t)\right]^{\frac{1}{2 p-1}}+C h(t) \xi^{\alpha}(t) E^{\alpha}(t) \\
& +c(\xi E)^{\alpha}(t)\left[-E^{\prime}(t)\right]^{\frac{2 m-2}{m}}
\end{aligned}
$$

Using (26), then (73) becomes

$$
\begin{aligned}
\xi^{\alpha+1}(t) E^{\alpha}(t) L^{\prime}(t) \leq & -\alpha_{1} \xi^{\alpha+1}(t) E^{\alpha+1}(t)+c(\xi E)^{\alpha}(t)\left[-E^{\prime}(t)\right]^{\frac{1}{2 p-1}}+C h(t) \xi^{\alpha}(t) E^{\alpha}(t) \\
& +c(\xi E)^{\alpha}(t)\left[-E^{\prime}(t)\right]^{\frac{2 m-2}{m}-\frac{1}{2 p-1}}\left[-E^{\prime}(t)\right]^{\frac{1}{2 p-1}} .
\end{aligned}
$$

Recalling Remark (4.2), we get

$$
\xi^{\alpha+1}(t) E^{\alpha}(t) L^{\prime}(t) \leq-\alpha_{1} \xi^{\alpha+1}(t) E^{\alpha+1}(t)+c(\xi E)^{\alpha}(t)\left[-E^{\prime}(t)\right]^{\frac{1}{2 p-1}}+C h(t) \xi^{\alpha}(t) E^{\alpha}(t) .
$$

Since $\alpha=2 p-2$, then we have

$$
\xi^{\alpha+1}(t) E^{\alpha}(t) L^{\prime}(t) \leq-\alpha_{1} \xi^{\alpha+1}(t) E^{\alpha+1}(t)+c(\xi E)^{\alpha}(t)\left[-E^{\prime}(t)\right]^{\frac{1}{\alpha+1}}+C h(t) \xi^{\alpha}(t) E^{\alpha}(t) .
$$

Now, repeating the same calculation as that in the proof of Theorem (4.1), we obtain (68). 
For the proof of (69), we multiply (25) by $\xi^{\alpha+1}(t) E^{\alpha}(t)$, use (17), recall the definition of $h(t)$ and Remark 4.1, then (73) becomes

$$
\begin{aligned}
\xi^{\alpha+1}(t) E^{\alpha}(t) L^{\prime}(t) \leq & -\alpha_{1} \xi^{\alpha+1}(t) E^{\alpha+1}(t)+c(\xi E)^{\alpha}(t)\left[-E^{\prime}(t)\right]^{\frac{1}{2 p-1}-\frac{2 m-2}{m}}\left[-E^{\prime}(t)\right]^{\frac{2 m-2}{m}} \\
& +C h(t) \xi^{\alpha}(t) E^{\alpha}(t)+c(\xi E)^{\alpha}(t)\left[-E^{\prime}(t)\right]^{\frac{2 m-2}{m}} .
\end{aligned}
$$

Using Remark (4.2), we get

$$
\xi^{\alpha+1}(t) E^{\alpha}(t) L^{\prime}(t) \leq-\alpha_{1} \xi^{\alpha+1}(t) E^{\alpha+1}(t)+c(\xi E)^{\alpha}(t)\left[-E^{\prime}(t)\right]^{\frac{2 m-2}{m}}+C h(t) \xi^{\alpha}(t) E^{\alpha}(t) .
$$

Since $\alpha=\frac{2-m}{2 m-2}$, then we have

$$
\xi^{\alpha+1}(t) E^{\alpha}(t) L^{\prime}(t) \leq-\alpha_{1} \xi^{\alpha+1}(t) E^{\alpha+1}(t)+c(\xi E)^{\alpha}(t)\left[-E^{\prime}(t)\right]^{\frac{1}{\alpha+1}}+C h(t) \xi^{\alpha}(t) E^{\alpha}(t) .
$$

Now, repeating the same calculation as that in the proof of Theorem (4.1), we can establish (69) and (71).

\section{Acknowledgements}

The authors thank KFUPM for its continuous support. This work is funded by KFUPM under Project \# SB181018.

\section{Funding}

This work is funded by KFUPM under Project (SB181018).

\section{Abbreviations}

Not applicable.

Availability of data and materials

Not applicable.

\section{Competing interests}

The authors declare that they have no competing interests.

\section{Authors' contributions}

We read and approved the final manuscript.

\section{Authors' information}

Not applicable.

\section{Publisher's Note}

Springer Nature remains neutral with regard to jurisdictional claims in published maps and institutional affiliations.

Received: 11 June 2019 Accepted: 13 August 2019 Published online: 28 August 2019

\section{References}

1. Cavalcanti, M.M., Domingos Cavalcanti, V.N., Soriano, J.A.: Exponential decay for the solution of semilinear viscoelastic wave equations with localized damping. Electron. J. Differ. Equ. 2002, 44 (2002)

2. Berrimi, S., Messaoudi, S.A.: Exponential decay of solutions to a viscoelastic equation with nonlinear localized damping. Electron. J. Differ. Equ. 2004, 88 (2004)

3. Fabrizio, M., Polidoro, S.: Asymptotic decay for some differential systems with fading memory. Appl. Anal. 81(6), 1245-1264 (2002)

4. Cavalcanti, M.M., Oquendo, H.P.: Frictional versus viscoelastic damping in a semilinear wave equation. SIAM J. Control Optim. 42(4), 1310-1324 (2003)

5. Muñoz Rivera, J.E.: Asymptotic behaviour in linear viscoelasticity. Q. Appl. Math. 52(4), 628-648 (1994)

6. Rivera, J.E.M., Lapa, E.C.: Decay rates of solutions of an anisotropic inhomogeneous n-dimensional viscoelastic equation with polynomially decaying kernels. Commun. Math. Phys. 177(3), 583-602 (1996)

7. Rivera, J.M., Lapa, E.C., Barreto, R.: Decay rates for viscoelastic plates with memory. J. Elast. 44(1), 61-87 (1996)

8. Rivera, J.E.M., Oquendo, H.P.: Exponential stability to a contact problem of partially viscoelastic materials. J. Elast. 63(2), 87-111 (2001)

9. Muñoz Rivera, J.E., Peres Salvatierra, A.: Asymptotic behaviour of the energy in partially viscoelastic materials. Q. Appl. Math. 59(3), 557-578 (2001) 
10. Pazoto, A.F., Bravo, J.V., Rivera, J.M.: Asymptotic stability of semigroups associated to linear weak dissipative systems Math. Comput. Model. 40(3-4), 387-392 (2004)

11. Rivera, J.E.M., Naso, M.G., Vegni, F.M.: Asymptotic behavior of the energy for a class of weakly dissipative second-order systems with memory. J. Math. Anal. Appl. 286(2), 692-704 (2003)

12. Hassan, J.H., Messaoudi, S.A.: General decay rate for a class of weakly dissipative second-order systems with memory. Math. Methods Appl. Sci. 42(8), 2842-2853 (2019)

13. Giorgi, C., Rivera, J.E.M., Pata, V.: Global attractors for a semilinear hyperbolic equation in viscoelasticity. J. Math. Anal. Appl. 260(1), 83-99 (2001)

14. Conti, M., Pata, V.: Weakly dissipative semilinear equations of viscoelasticity. Commun. Pure Appl. Anal. 4(4), 705 (2005)

15. Appleby, J.A., Fabrizio, M., Lazzari, B., Reynolds, D.W.: On exponential asymptotic stability in linear viscoelasticity. Math. Models Methods Appl. Sci. 16(10), 1677-1694 (2006)

16. Pata, V: Stability and exponential stability in linear viscoelasticity. Milan J. Math. 77(1), 333 (2009)

17. Guesmia, A.: Asymptotic stability of abstract dissipative systems with infinite memory. J. Math. Anal. Appl. 382(2), 748-760 (2011)

18. Guesmia, A., Messaoudi, S.A.: A general decay result for a viscoelastic equation in the presence of past and finite history memories. Nonlinear Anal., Real World Appl. 13(1), 476-485 (2012)

19. Messaoudi, S.A., Al-Gharabli, M.M.: A general stability result for a nonlinear wave equation with infinite memory. Appl. Math. Lett. 26(11), 1082-1086 (2013)

20. Cavalcanti, M., Guesmia, A., et al.: General decay rates of solutions to a nonlinear wave equation with boundary condition of memory type. Differ. Integral Equ. 18(5), 583-600 (2005)

21. Youkana, A.: Stability of an abstract system with infinite history (2018). arXiv:1805.07964

22. Al-Gharabli, M.M.: A general decay result of a viscoelastic equation with infinite history and nonlinear damping. Appl. Anal. 97(3), 382-399 (2018)

23. Belhannache, F., Algharabli, M.M., Messaoudi, S.A.: Asymptotic stability for a viscoelastic equation with nonlinear damping and very general type of relaxation functions. J. Dyn. Control Syst. 1-23 (2019)

24. Messaoudi, S.A., Al-Khulaifi, W.: General and optimal decay for a viscoelastic equation with boundary feedback. Topol. Methods Nonlinear Anal. 51(2), 413-427 (2018)

25. Guesmia, A., Messaoudi, S.: A new approach to the stability of an abstract system in the presence of infinite history. J. Math. Anal. Appl. 416(1), 212-228 (2014)

\section{Submit your manuscript to a SpringerOpen ${ }^{\circ}$ journal and benefit from:}

- Convenient online submission

- Rigorous peer review

- Open access: articles freely available online

- High visibility within the field

- Retaining the copyright to your article

Submit your next manuscript at $\boldsymbol{~ s p r i n g e r o p e n . c o m ~}$ 